\title{
Les précipitations exceptionnelles des 3-4 octobre 1960 sur le nord-ouest du Massif Central
}

\author{
The exceptional precipitation \\ recorded in the north-western Central Massif \\ on the 3rd and 4th October 1960
}

\author{
par P. GUIILOT
}

CHEF DU SERVICE HYDROMÉTÉOROLOGIQUE DE LA DIVISION TECHNIQUE GÉNÉRALE PRODUCTION HYDRAULIQUE, ÉLECTRICITÉ DE FRANCE A GRENOBLE

\begin{abstract}
Isohyètes en forme d'ellipses concentriques dont le noyait, supérieur à $200 \mathrm{~mm}$, couvre les monts des Monédières et le plateau de Millevaches et dont le grand axe $(100 \mathrm{~mm})$ s'allonge de Gourdon jusqu'au nord de Montluçon, laissant en marge les grands reliefs condensateurs habituels : Sancy, Cantal, Aubrac $(50 \mathrm{~mm})$.

Chronologie du phénomène: synchronisme $d u$ blocage du front pluvieux initial sur la rive droite de la Dordogne avec les intensités maximales sur le plateau de Millevaches, $20 \dot{a}$ $30 \mathrm{~mm} / \mathrm{h} e u r e$, élevées pour la région bien que modestes vis-à-vis des régions méditerranéennes.
\end{abstract}

Ajustement d'une loi de Gumbel ou de Fréchet situant la durée du retour d'un tel phénomène à plusieurs siècles, voire au millénaire.

\begin{abstract}
Concentric elliptical isohyets with a central core (over $200 \mathrm{mms}$ ) covering the Monédières hills and the Millevaches plateau, with a major axis (100 mms) running from Gourdon to a point north of Montluçon, missing the usual large hilly condensating areas, i.e. Sancy, Cantal and Aubrac (50 mms).

The initial rain front was held up at the right bank of the river Dordogne just at the time maximum rainfall intensities occurred on the Millevaches plateau $(20-30 \mathrm{mms} /$ hour $)$. Though not very high by Mediterranean standards, these values are nevertheless considerable for the area concerned.

Adjustment of a Gumbel or Fréchet law, according to which such conditions only recur at intervals of several centuries or every thousand years.
\end{abstract}

Dans l'après-midi du 3 octobre 1960, des pluies d'une abondance presque inconcevable dans cette région, se sont abattues sur le haut Limousin. Les crues des rivières qui descendent du plateau de Millevaches, en particulier la Creuse au nord, la Corrèze au sud, ont dévasté les villes d'Aubusson, Tulle et Brive.

L'objet de cette communication est simplement de décrire le phénomène «précipitations 》 d'après les données du réseau d'observation équipé et exploité par Electricité de France, Service de la Production Hydraulique. 


\section{A. - CARTE DES ISOHYÈTES}

La figure 1 est une carte à petite échelle, sur l'ensemble du Massif Central, des isohyètes des précipitations du $3-4$ octobre 1960 , et la figure 2 une vue plus détaillée, limitée au quart nordouest.

\section{Validité de ces mesures :}

Bien que la majeure partie des précipitations ait eu lieu en une vingtaine d'heures, soit entre 8 heures le 3 et 4 heures le 4 , nous avons été conduits à additionner les relevés pluviométriques des 3,4 et 5 au matin, les pluies ayant commencé dans l'ouest de la région vers 6 heures du matin le 3 et s'étant terminées dans le milieu de la journée du 4; nous présentons, néanmoins, en figure 3 , les isohyètes du seul relevé du 4 au matin, qui rappellent beaucoup celles de la figure 2 moyennant un abattement variant de 10 à $30 \mathrm{~mm}$.

Dans plusieurs stations du plateau de Mille-

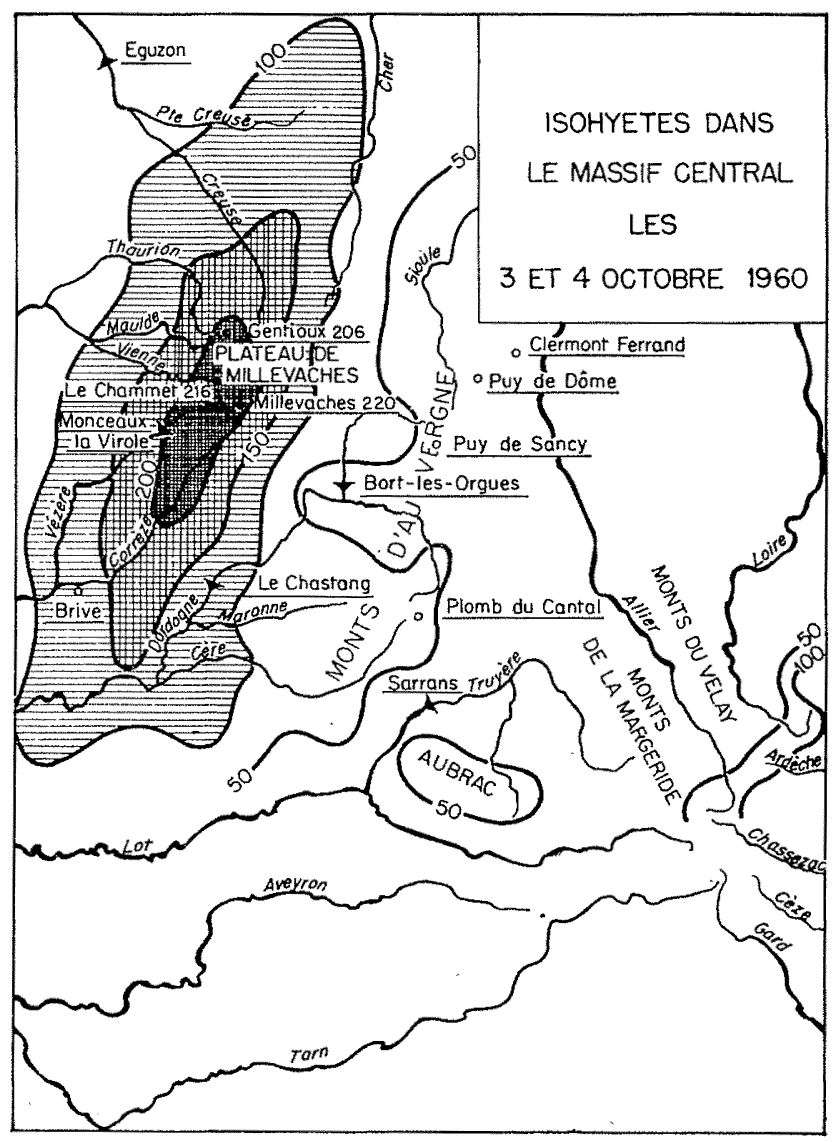

Fig. 1 vaches, les mesures du 4 sont proches de la capacité maximale des pluviomètres, ce qui pourrait laisser craindre qu'il y ait eu écrêtement : l'existence d'enregistreurs étalonnés à Saint-Merdles-Oussines et au Chammet, qui ont bien fonctionné, et qui recoupent à 2 ou $3 \%$ près la mesure faite avec le pluviomètre adjacent (1), la certitude que plusieurs observateurs et en particulier celui de Millevaches ont effectué un relevé intermédiaire dans l'après-midi du 3, permettent d'accorder assez de crédit aux valeurs inscrites dans la région du maximum. L'intérêt éveillé chez nos correspondants par un phénomène aussi extrême, est un facteur supplémentaire favorable à la qualité des observations.

$2^{\circ}$ On note, compte tenu d'une erreur aléatoire possible de l'ordre de $10 \mathrm{~mm}$, la bonne cohérence des mesures et la régularité assez remarquable des isohyètes qui affectent dans l'ensemble la forme d'ellipses allongées SSWNNE, autour du maximum de $220 \mathrm{~mm}$ à Millevaches, qui joue le rôle de pôle d'homothétie: la $200 \mathrm{~mm}$, longue de $60 \mathrm{~km}$ et large de $20 \mathrm{~km}$ couvre toute la vallée de la Corrèze en amont de Tulle, le bassin de la Vézère à Bugeat, celui de la Vienne à Servières et atteint la source du Thaurion.

La $100 \mathrm{~mm}$, longue de $250 \mathrm{~km}$ et large de 50 à 60, s'étend depuis Gourdon jusqu'au nord de Montluçon.

On note également que le gradient pluviométrique est environ 2,5 fois plus fort sur le versant est de la dorsale pluviométrique que sur le versant ouest; vers l'est la distance de Millevaches à l'isohyète $60 \mathrm{~mm}$ est de $30 \mathrm{~km}$, contre $75 \mathrm{~km}$ vers l'ouest.

Parmi les conséquences de cette rapide décroissance vers l'est, on voit que les trois affluents rive droite de la Dordogne : Diège, Triouzoune et Luzège, ont reçu, près de leur confluent avec la Dordogne, une lame d'eau environ trois fois plus faible que dans la partie amont de leur bassin; on voit aussi combien la Tardes et le Cher en amont de leur confluent ont été inégalement traités, la Tardes recevant plus de deux fois plus que le Cher.

$3^{\circ}$ Mais ce qui est sans doute le plus extraordinaire, plus même peut-être que l'abondance des pluies, c'est que les grands reliefs conden-

(1) Résultats comparés au pluviomètre et au pluviographe :

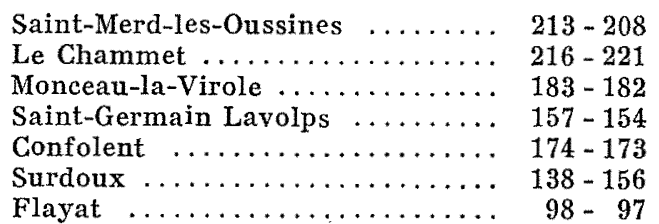




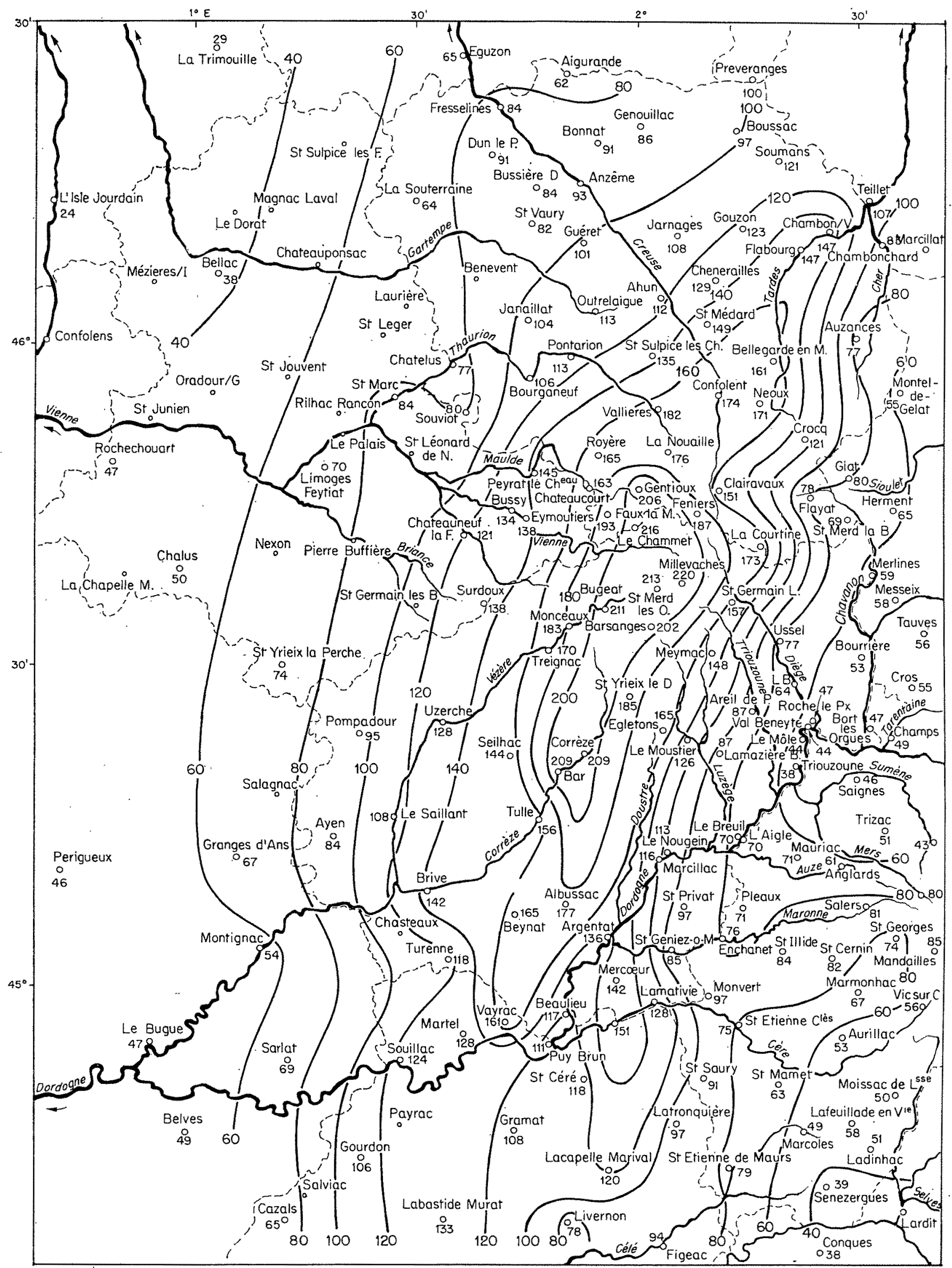

FIG. 2 


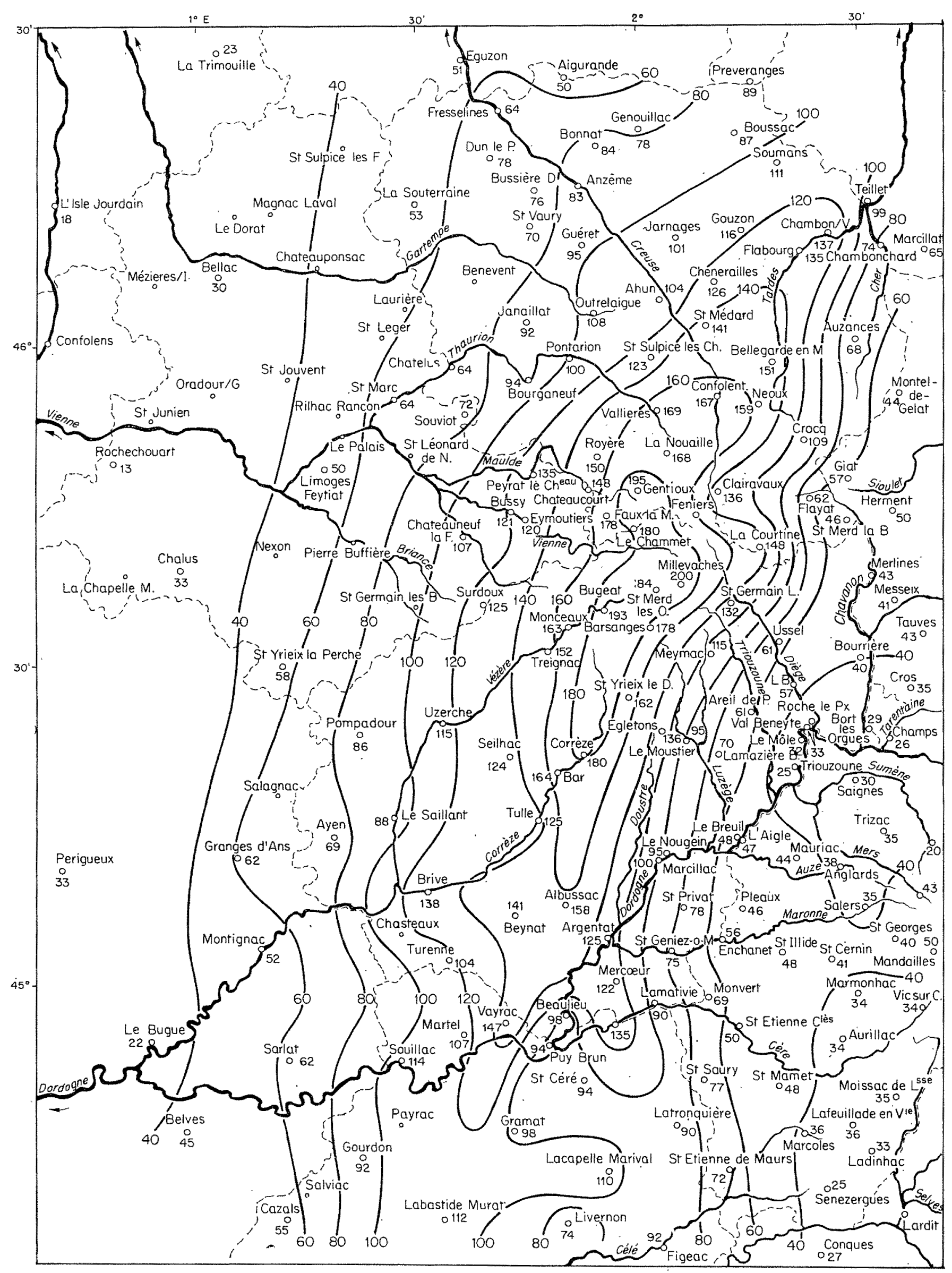

FIG. 3 
sateurs habituels de l'ouest du Massif Central, ceux dont l'effet orographique, à chaque passage de perturbation atlantique, apparaît comme une loi quand on consulte les collections de cartes d'épisodes pluvieux, sont restés cette fois-ci nettement en retrait, comme non concernés par le conflit qui se déroulait à quelques dizaines de kilomètres devant eux, distance qui, à la vitesse habituelle de déplacement des perturbations, est franchie en 1 heure ou moins; souvent même, les précipitations débutent sur ces reliefs avant le passage du front proprement dit.

\section{B. - HORAIRE ET INTENSITÉ}

Sur la carte de la figure 4 est portée l'heure du début des précipitations continues dans la journée du 3 octobre : on voit qu'après avoir débuté vers 6 heures sur une ligne EguzonMontignac, la pluie gagnait en moins de 2 heures le plateau de Millevaches et couvrait, dès 9 heures tout le bassin du Cher à Teillet et de la Creuse à Eguzon, atteignant Tulle et Brive, mais ne débordait que peu dans le bassin de la Dordo-

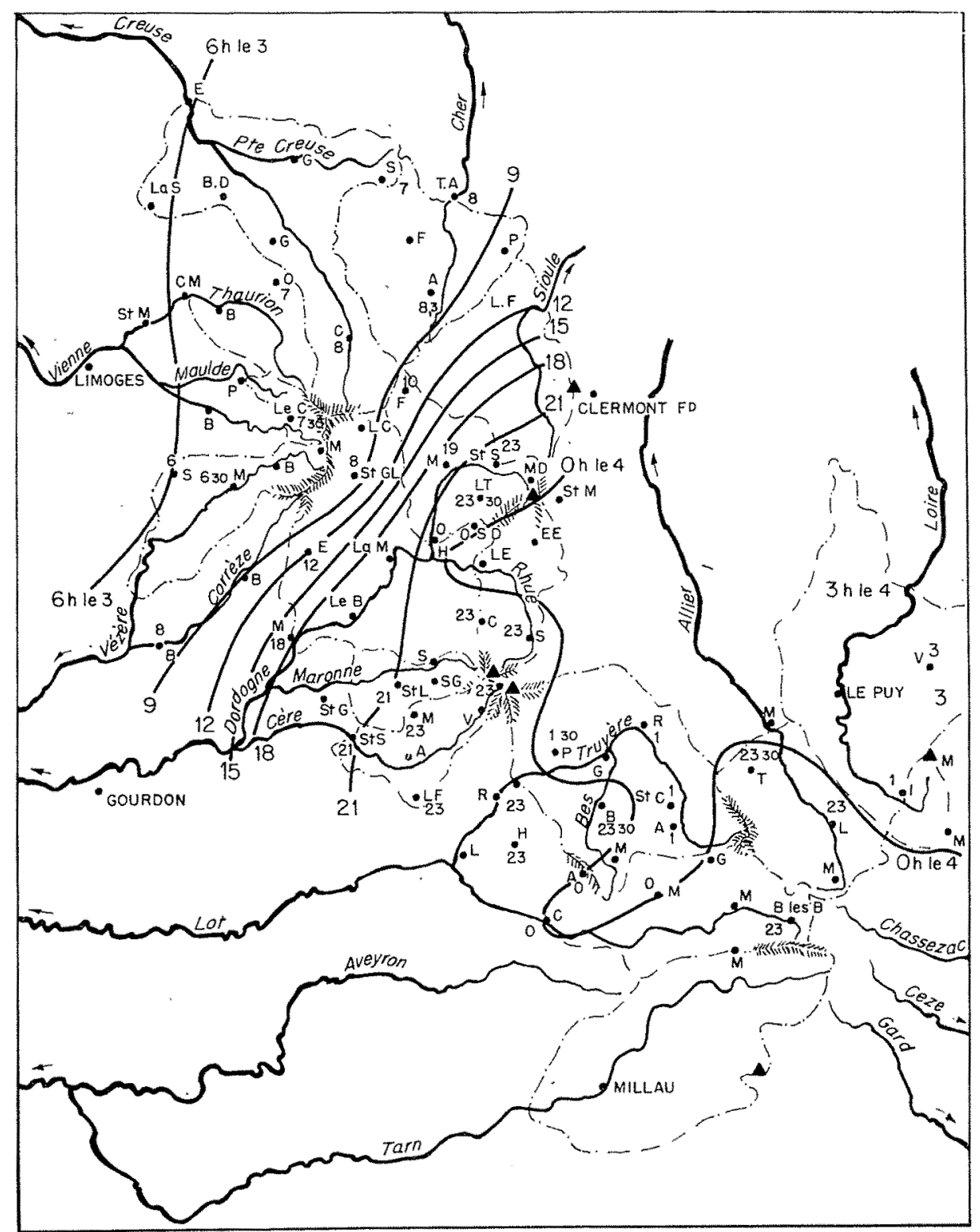

Fig. 4

Carte des précipitations des 3 et 4 octobre 1960 . Isochrones du début des précipitations continues (d'après les pluviogrammes; heures légales). 


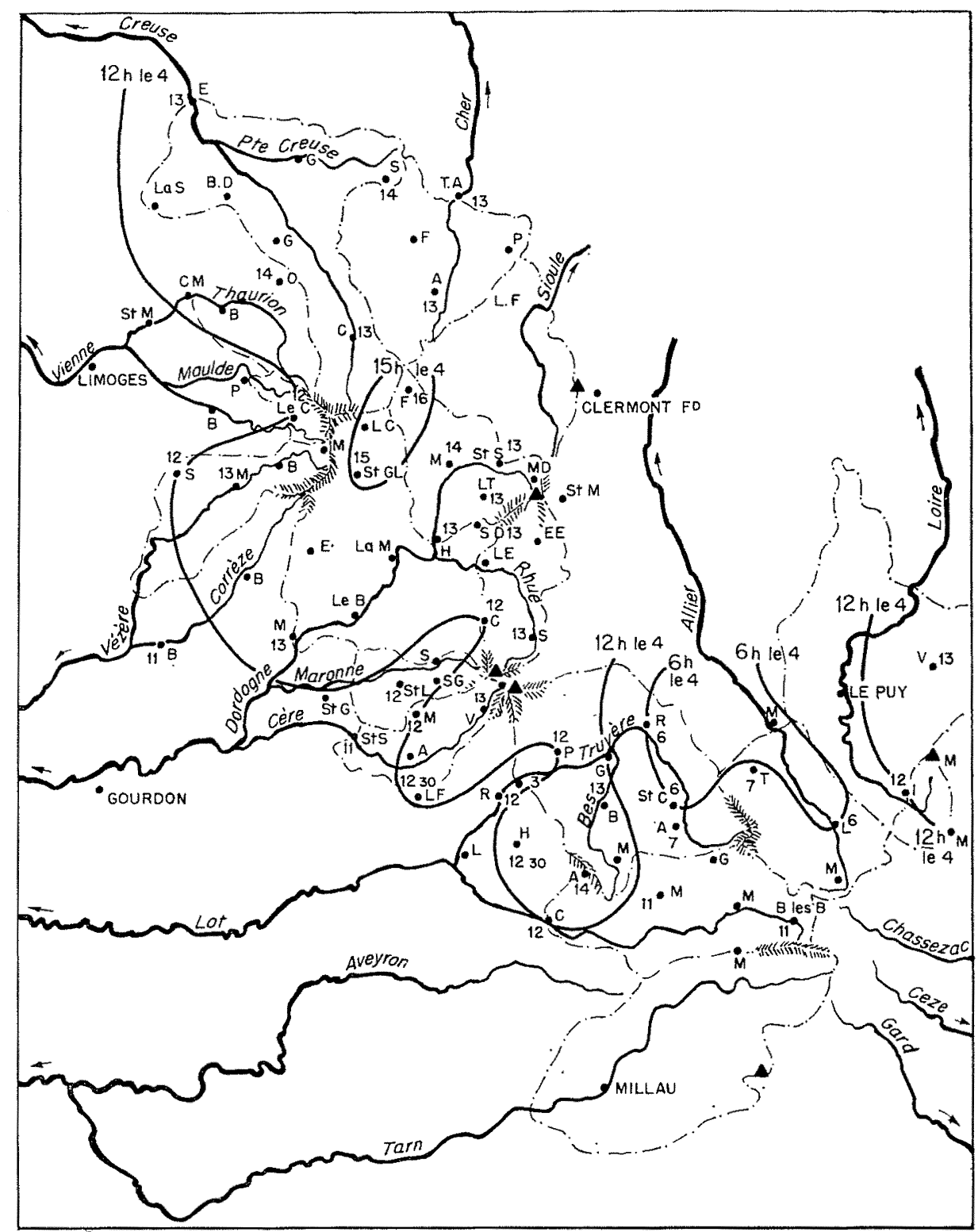

FIG. 5

Carte des précipitations des 3 et 4 octobre 1960.

Isochrones de la fin des précipitations continues (d'après les pluviogrammes; heures légales, le 4).

gne à Argentat par le haut des vallées des Diège, Triouzoune et Luzège. Dès lors, pendant tout l'après-midi et la soirée du 3 , on constate que la progression vers l'est est très lente : la vallée de la Dordogne n'est franchie qu'entre 19 et 21 heures et la pluie intense et continue ne dehute qu'entre 23 heures et 24 heures sur la ligne Sancy-Cantal-Aubrac. Elle n'y dure que 3 à 4 heures, donnant 15 à $30 \mathrm{~mm}$, puis elle est suivie d'une traîne d'averses entrecoupées d'accalmies, jusque vers 12 heures le 4 . En somme, sur les grands reliefs, la perturbation des 3-4 octobre s'est déroulée comme un passage classique de front froid vigoureux, mais non exceptionnel; il s'en présente plusieurs par an de cette taille. La figure 5 indique l'horaire de la fin des pluies dans la journée du 4.

C'est dans l'après-midi du 3 , précisément à l'heure où la progression vers l'est paraìt rencontrer le plus de résistance, que se produit, sur le plateau de Millevaches, le paroxisme d'intensité. Le graphique 6 donne les hyétogrammes heure par heure des enregistreurs de Saint-Merdles-Oussines et du Chammet. On voit que cette 
intensité a dépassé $20 \mathrm{~mm}$ en une heure et l'examen attentif du diagramme original de SaintMerd-les-Oussines permet d'avancer qu'il est tombé $20 \mathrm{~mm}$ en 40 minutes entre $16 \mathrm{~h} 40$ et $17 \mathrm{~h} 20$, soit un demi-millimètre à la minute, et $26 \mathrm{~mm}$ en 1 heure entre $16 \mathrm{~h} 30$ et $17 \mathrm{~h} 30$. Il paraît évidemment hasardeux d'extraire de diagrammes à déroulement hebdomadaire, dont la vitesse est de $2,4 \mathrm{~mm}$ à l'heure $(400 \mathrm{~mm}$ en 168 heures), des intensités sur des intervalles de temps notablement plus courts qu'une heure, mais la comparaison détaillée des enregistrements permet d'affirmer que c'est bien à SaintMerd-les-Oussines qu'ont été observées les intensités les plus fortes; l'aspect régulier du trait d'enregistrement et l'apparente continuité de sa pente laissent penser que l'intensité n'a pas dû varier très rapidement et que, même sur des intervalles de temps de quelques minutes, le demi-millimètre/minute n'a sans doute pas été beaucoup dépassé. Nulle part n'a été observé plus de $40 \mathrm{~mm}$ en 2 heures (cette valeur a été seulement atteinte. à Saint-Merd-les-Oussines entre $16 \mathrm{~h} 30$ et $18 \mathrm{~h} 30$ ni plus de $75 \mathrm{~mm}$ en 4 heures (observé à Saint-Merd-les-Oussines entre 16 heures et 20 heures).

On est donc encore loin des intensités connues dans les régions cévenoles où l'on sait qu'ont été observées $210 \mathrm{~mm}$ en 4 heures à La Rouvière de Cros le 30 septembre 1958, avec des intensités instantanées dépassant donc largement le millimètre à la minute (mais qui avaient été laminées par l'orifice de contrôle du pluviographe).

Il ne faudrait pas pourtant en déduire que le millimètre/minute est impossible sur le versant atlantique du Massif central : nous avons observé $30 \mathrm{~mm}$ en 30 minutes à Bort, mais sous un orage très local, limité sans doute à quelques $\mathrm{km}^{2}$, tandis que, le 3 octobre 1960 , plusieurs milliers de $\mathrm{km}^{2}$ étaient simultanément arrosés.

\section{***}

\section{C. - FREQUENCE}

Pour tenter de comparer l'événement des 3-4 octobre 1960 aux précipitations les plus fortes connues dans le passé de cette région, nous n'avons pas voulu retenir les valeurs journalières notées par une station isolée : la durée d'une forte perturbation pluvieuse est généralement une fraction importante d'une journée et peut même, comme ce fut précisément le cas des $3-4$ octobre 1960 dépasser quelque peu 24 heures; aussi le découpage chronologique quotidien ris-

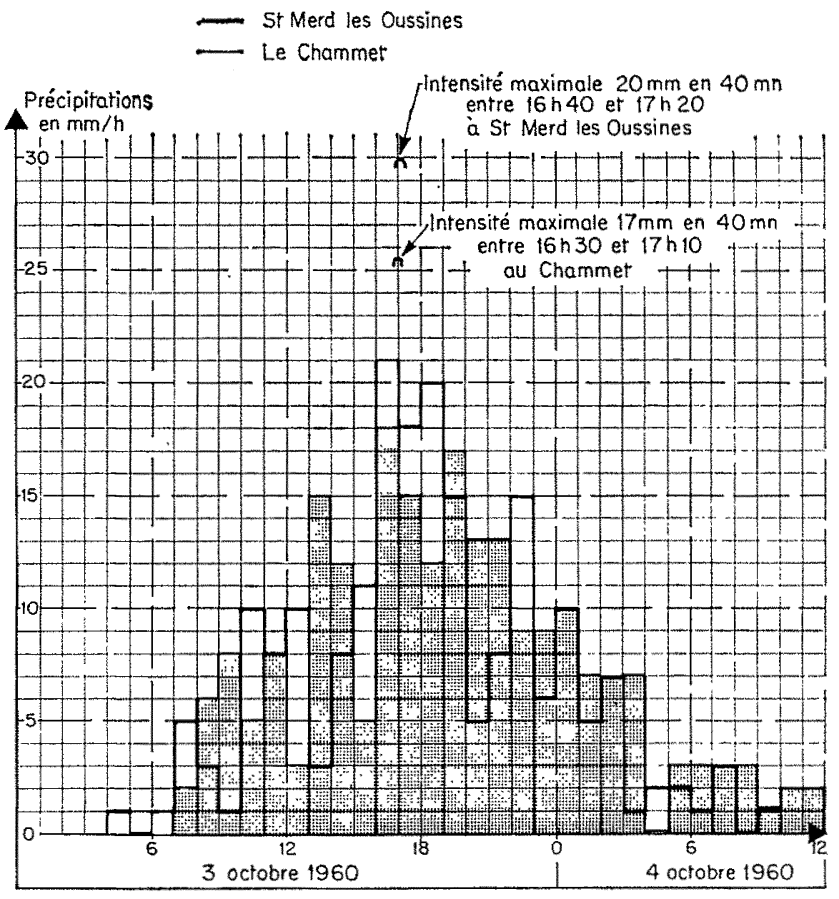

FIG. 6

Pluies des 3 et 4 octobre 1960. Intensité horaire sur le plateau de Millevaches.

que-t-il d'amputer le phénomène naturel et d'altérer gravement sa loi de distribution; si les 3-4 octobre, on s'est trouvé dans le cas favorable où l'essentiel de la précipitation est tombé dans l'intervalle de 24 heures précédant le relevé du 4 , il n'y a aucune raison qu'il en soit toujours de même. C'est pourquoi nous croyons déjà nettement plus significatif d'examiner la valeur maximale prise par deux relevés journaliers consécutifs, faute de disposer d'enregistrements horaires remontant suffisamment loin dans le passé.

Nous croyons également préférable de ne pas considérer une station unique, mais la moyenne de plusieurs stations de la région, séparées par quelques dizaines de kilomètres, ceci afin d'éliminer des orages tout à fait locaux.

En définitive, compte tenu des ressources en données climatologiques anciennes, malheureusement fort limitées (2), nous avons recherché les valeurs maximales annuelles de la somme de deux précipitations journalières consécutives et pour la moyenne des trois stations Brive-TulleUssel sur la série des quarante années 1920 à 1959.

Le résultat de cette comparaison est illustré

(2) A cet égard, la Corrèze avec cinq stations qui ont fonctionné tant bien que mal depuis 1920 , n'est pas parmi les départements français les plus défavorisés. 


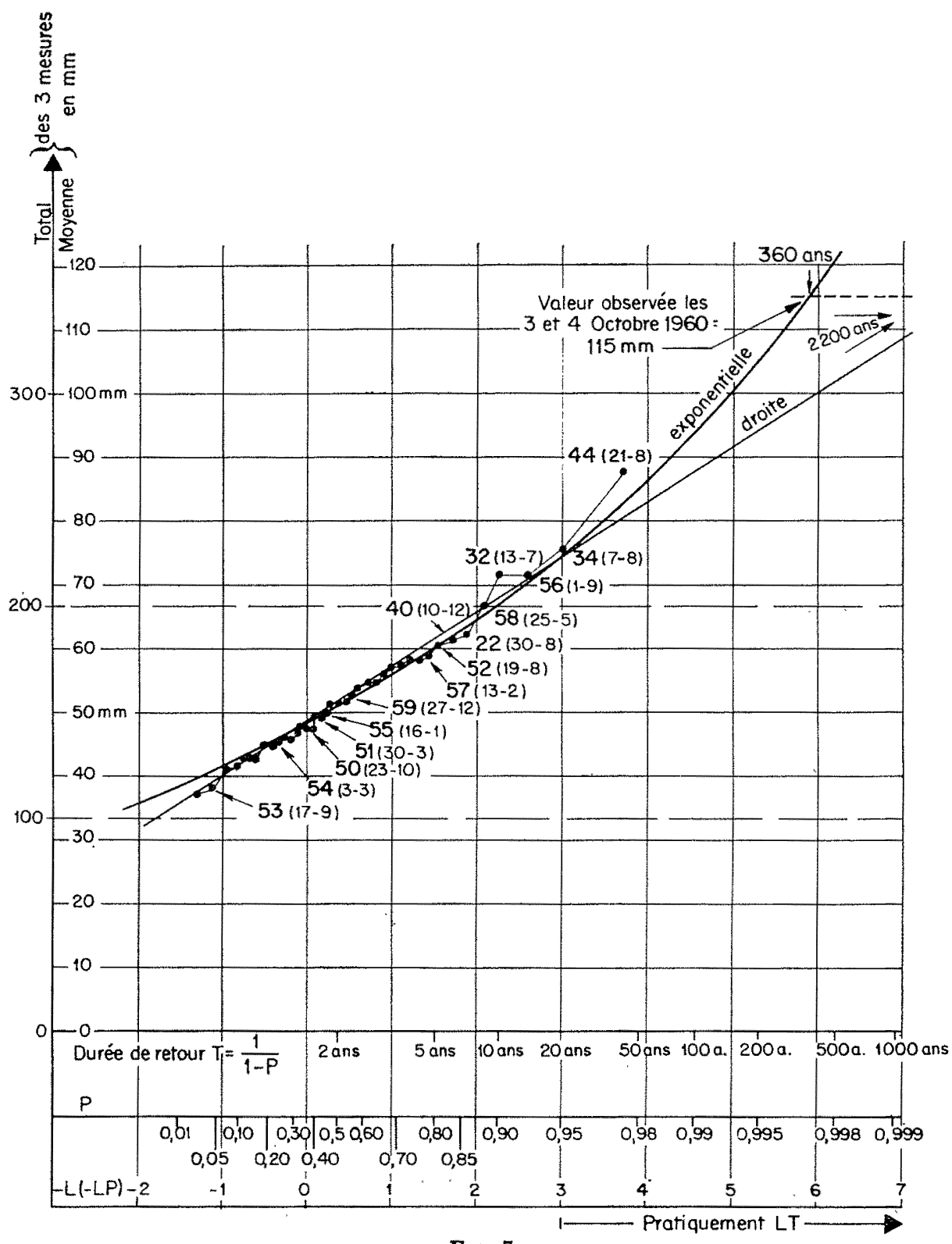

FrG. 7

Maximum annuel de précipitations en 48 heures. Moyenne de trois stations : Brive, Tulle, Ussel.

par le graphique numéro 7 où apparaissent en ordonnée les quarante valeurs maximales annuelles, dont la moyenne est de $53,3 \mathrm{~mm}$ et l'écarttype $10,7 \mathrm{~mm}$ : on voit qu'on a guère de raison de choisir l'un plutôt que l'autre des ajustements de Gumbel (droite) ou de Fréchet (exponentielle) qui sont tous les deux satisfaisants pour les valeurs faibles et moyennes (on pourrait tout aussi bien ajuster une parabole de degré quelconque), mais que la dispersion des valeurs supérieures incite à se méfier de toute extrapolation : on peut dire cependant - et dans ce domaine, la règle est d'avoir à se contenter d'ordres de grandeur - qu'il paraît peu probable que la durée de retour d'une précipitation de $115 \mathrm{~mm}$ soit inférieure à un siècle, et qu'elle est vraisemblablement de plusieurs siècles.

Il faut remarquer cependant que Brive, Tulle et Ussel sont situés en marge de la région des intensités maximales des $3-4$ octobre 1960 ; nous les avons utilisés faute d'avoir de longues séries d'observations sur le plateau de Millevaches proprement dit.

Les $200 \mathrm{mim}$ tombés sur le plateau de Millevaches constituent sans doute un événement encore plus improbable, même si l'on tient compte de ce que les pluies y sont en moyenne plus fortes qu'à Brive-Ussel-Tulle. 


\section{I S C US S I O N}

Président: M. de Rouville

M. le Président demande à M. Gurllot s'il a quelque chose à ajouter à ce qui a été dit par M. JAcQueT.

M. Guillot pense que M. JACQUer a très rapidement et très bien résumé son rapport.

M. le Président demande où se trouvent les monts de Monédières.

M. GuILLot précise que ces monts se trouvent au sudouest du plateau de Millevaches, et qu'ils séparent la Vézère de la Corrèze.

C'est de ces monts qu'a coulé la Vimbelle dont le débit spécifique a été considérable.

M. Remenieras pense que le débit spécifique observé, déjà considérable par rapport au bassin versant, a été souvent dépassé sur de petits bassins versants du Massif Central. Les débits spécifiques ont-ils diminué avec l'augmentation de la superficie des bassins?

M. JAcQuET répond que la Vimbelle a contribué à faire crôtre le débit spécifique de l'amont vers l'aval sur la Corrèze, alors que, généralement, les débits spécifiques allaient en décroissant de l'amont vers l'aval.

M. Gullor signale, au sujet des moyens de transmission pour les prévisions des crues, que le seau du pluviomètre est un instrument peu pratique et démodé et que l'on emploie maintenant des compteurs d'impulsions qui sont branchés sur les pluviographes et qui sont devant les yeux des observateurs et à côté du téléphone: c'est un premier stade avant d'avoir la radio. En outre, la connaissance de la situation météorologique et une bonne habitude des effets orographiques de la région sont indispensables pour mener à bien les enquêtes sur les pluies.

M. le Président estime que, dans les régions de pénéplaine, les points névralgiques sont moins définis que dans les Cévennes et les Pyrénées.

M. Guillot pense qu'en plaine il n'y a pas de point névralgique lorsque c'est une siluation frontale, un front bien régulier qui approche: la ligne de pluie s'avance de façon régulière et rectiligne. Par contre, en cas de situation orageuse diffuse, les taches de pluie sont intenses, étroitement localisées et difficilement prévisibles. Le radar est alors fort précieux.

A Ia lumière de ce qu'il a pu voir récemment aux Etats-Unis, M. Remenieras pense que le radar météorologique peut apporter dans un avenir prochain de grandes facilités dans ce domaine. Sur l'écran de cet appareil apparaissent des taches dont la luminosité est inversement proportionnelle à l'intensité des précipitations. En superposant des clichés photographiques de cet écran (pris toutes les minutes ou toutes les cinq minutes), on peut obtenir, au moyen d'un opacimètre photométrique, une évaluation approximative de l'intensité moyenne ou de la hauteur totale des précipitations tombées sur une zone déterminée dans un intervalle de temps quelconque. L'étalonnage de l'opacimètre est fait d'après les indications d'un ou plusieurs pluviomètres à transmission radio existant dans la zone couverte par le radar.

M. le Président pense qu'avec un peu d'habitude, on arrive à voir si la tache correspond à une pluie diluvienne ou une pluie moins intense.

M. GunLLor précise que le radar ne donne que l'intensité instantanée de la pluie, il n'intègre pas. Les Américains essayent, en superposant les images données par le radar, d'avoir une idée de l'isohyète des dernières heures.

M. Guillor ajoute qu'un service américain possède, à la Station centrale d'observations, un appareil qui superpose sur l'écran radar l'image de la pluie instantanée et des réglettes dont la longueur est proportionnelle à la hauteur d'eau tombée dans les pluviographes du réseau au cours des dernières heures.

M. le Président pense qu'il est plus intéressant de savoir l'avenir d'une averse que de connaitre son passé, parce que selon que l'averse dure 3 ou 8 heures, elle produit des résultats différents. Est-ce que la météorologie permet de dire si une pluie intense qui commence va durer un certain temps ou si elle va cesser? C'est cela qui fait les grosses crues.

M. Fontaine répond qu'on peut le faire sur 24 heures: tout au moins on peut espérer faire des progrès pour une prévision à plus bref délai.

M. le Président estime qu'il faudrait essayer de poursuivre des recherches dans ce sens.

M. Gurllot précise que, pour en revenir à la question de l'organisation matérielle, Iorsqu'il s'agit d'endroits témoins très intéressants, on peut mettre un équipement onéreux : par exemple, un pluviophone qui répond tout seul au téléphone quand on l'interroge. Cet appareil indique la hauteur d'eau existant dans le pluviomètre à l'instant où on l'interroge et l'enregistrement de la hauteur d'eau dans le passé.

M. le Président remercie M. GullLot. 